\title{
Systematic Review \\ Mindfulness-Based Interventions for Physical and Psychological Wellbeing in Cardiovascular Diseases: A Systematic Review and Meta-Analysis
}

\author{
Flavia Marino ${ }^{1,+}$, Chiara Failla ${ }^{1,2,+}$, Cristina Carrozza ${ }^{1,+}$, Maria Ciminata ${ }^{3}$, Paola Chilà ${ }^{1}$, Roberta Minutoli ${ }^{1}$, \\ Sara Genovese ${ }^{1}$, Alfio Puglisi ${ }^{1}$, Antonino A. Arnao ${ }^{1}$, Gennaro Tartarisco ${ }^{1}$ (D), Flavio Corpina ${ }^{1,4}$, \\ Sebastiano Gangemi ${ }^{5}$, Liliana Ruta ${ }^{1}$, Antonio Cerasa ${ }^{6,7}$, David Vagni ${ }^{1}$ and Giovanni Pioggia ${ }^{1, * \text { (D) }}$
}

Citation: Marino, F.; Failla, C.; Carrozza, C.; Ciminata, M.; Chilà, P.; Minutoli, R.; Genovese, S.; Puglisi, A.; Arnao, A.A.; Tartarisco, G.; et al. Mindfulness-Based Interventions for Physical and Psychological Wellbeing in Cardiovascular Diseases: A Systematic Review and Meta-Analysis. Brain Sci. 2021, 11, 727. https://doi.org/10.3390/ brainsci11060727

Academic Editor: Dona E. Locke

Received: 28 April 2021

Accepted: 24 May 2021

Published: 29 May 2021

Publisher's Note: MDPI stays neutral with regard to jurisdictional claims in published maps and institutional affiliations.

Copyright: (c) 2021 by the authors Licensee MDPI, Basel, Switzerland. This article is an open access article distributed under the terms and conditions of the Creative Commons Attribution (CC BY) license (https:/ / creativecommons.org/licenses/by/ $4.0 /)$.
1 Institute for Biomedical Research and Innovation (IRIB), National Research Council of Italy (CNR), 98164 Messina, Italy; flavia.marino@cnr.it (F.M.); chiara.failla@irib.cnr.it (C.F.); cristina.carrozza@irib.cnr.it (C.C.); paola.chila@irib.cnr.it (P.C.); roberta.minutoli@irib.cnr.it (R.M.); sara.genovese@cnr.it (S.G.); alfio.puglisi@irib.cnr.it (A.P.); antoninoandrea.arnao@cnr.it (A.A.A.); gennaro.tartarisco@cnr.it (G.T.); fcorpina@cotmessina.it (F.C.); liliana.ruta@cnr.it (L.R.); david.vagni@irib.cnr.it (D.V.)

2 Classical Linguistic Studies and Education Department, Kore University of Enna, 94100 Enna, Italy

3 Departamento de Estudios Románicos, Franceses, Italianos y Traducción (ERFITEI), Universidad Complutense Madrid, 28040 Madrid, Spain; mciminat@ucm.es

4 Multi-Specialist Clinical Institute for Orthopaedic Trauma Care (COT), 98124 Messina, Italy

5 Operative Unit of Allergy and Clinical Immunology, Department of Clinical and Experimental Medicine, School of Allergy and Clinical Immunology, University of Messina, 98125 Messina, Italy; gangemis@unime.it

6 Institute for Biomedical Research and Innovation (IRIB), National Research Council of Italy (CNR), 87050 Mangone, Italy; antonio.cerasa@cnr.it

7 S'Anna Institute, Research in Advanced Neurorehabilitation (RAN), 88900 Crotone, Italy

* Correspondence: giovanni.pioggia@cnr.it

+ These authors contributed equally to the study.

Abstract: Background: Recently, there has been an increased interest in the efficacy of mindfulnessbased interventions (MBI) for people with cardiovascular diseases (CVD), although the exact beneficial effects remain unclear. Methods: This review aims to establish the role of MBI in the management of wellbeing for patients with CVD. Seventeen articles have been included in this systematic synthesis of the literature and eleven in the meta-analysis. Results: Considering physical (i.e., heart rate, blood pressure) and psychological outcomes (i.e., depression, anxiety, stress, styles of coping), the vast majority of studies confirmed that MBI has a positive influence on coping with psychological risk factors, also improving physiological fitness. Random-effects meta-analysis models suggested a moderate-to-large effect size in reducing anxiety, depression, stress, and systolic blood pressure. Conclusions: Although a high heterogeneity was observed in the methodological approaches, scientific literature confirmed that MBI can now be translated into a first-line intervention tool for improving physical and psychological wellbeing in CVD patients.

Keywords: mindfulness; cardiovascular diseases; psychological outcome; physical outcome; systematic review

\section{Introduction}

Cardiovascular disease (CVD) is among the leading causes of disability and mortality globally [1]. It is well known that this disorder is associated with a particular lifestyle, such as smoking, incorrect feeding, hyperlipidemia, hyperglycemia, hypertension, obesity, alcohol intake, and diabetes [2]. Furthermore, psychosocial stress is widely recognized as one of the main contributors to all these risk factors; therefore, behavioral approaches to primary and secondary prevention are gaining research support. According to the World 
Health Organization (WHO) [3], actions aimed at reducing these major risk factors can drastically impact disability and death from CVD.

Mindfulness-based interventions (MBI) have been proposed as an efficient and easily applicable additional complementary treatment for reducing psychological stress in the management of various physical and mental health conditions [4,5]. MBI generally refers to practices that cultivate awareness and require paying attention to the present moment [6]. Mindfulness training strengthens metacognitive awareness (self-reflective capacity to monitor mental experience), allowing participants to shift their perspective ("reperceiving") and reduce emotional reactivity [7]. The two main practices of MBI are Mindfulness-Based Stress Reduction (MBSR) and Mindfulness-Based Cognitive Therapy (MBCT). MBSR has been used since 1979 as a training vehicle for the relief of pain and distress in people with chronic health problems [8]. In general, this program includes 8 weekly meetings of 1-2 $\mathrm{h}$ approximately and 8 final hours of a full-day retreat $[9,10]$. MBSR has been found to have positive effects on pain, anxiety, and stress in people with chronic disorders such as fibromyalgia, cancer, arthritis, and coronary artery disease [11-13]. On the other hand, MBCT was initially developed as a relapse prevention treatment for those with a high risk of recurring depression, but it has further been adapted to a range of different clinical realms. This protocol teaches the subject to move away from ruminative mental patterns typical of depression and to adopt a new perspective in which thoughts and feelings are considered transitory rather than objective representations of reality [14].

To manage physical and psychological symptoms in CVD patients, meditative practices have been proposed, in addition to conventional medical interventions, as an effective approach for reducing clinical complications in CVD patients $[15,16]$. However, some inconsistent findings have been reported. Indeed, some studies have found that patients with CVD appear to benefit only psychologically from mindfulness interventions [15], whereas others suggest possible benefits also for physical risk factors, such as blood pressure, obesity, and smoking [2]. Currently, few studies have shown combined physical and psychological positive effects [17], while the vast majority described mixed results [15].

For this reason, we sought to perform a new synthesis of literature aimed at reexamining the question of whether there is sufficient evidence to conclude that MBI can be included in clinical recommendations for primary and secondary prevention of CVD.

\section{Materials and Methods}

This review was planned and conducted in accordance with Preferred Reporting Items for Systematic Reviews and Meta-Analyses (PRISMA) guidelines [18]. Articles published between 2003 and 2019 were reported using electronic bibliographic databases such as PubMed, Science Direct, and Google Scholar. To improve the search strategy, keywords including "text words" and MeSH were used. The search terms incorporated the following keywords: "mindfulness, meditative practices, cardiovascular diseases, heart failure, congestive heart failure, coronary heart disease, cardiovascular abnormalities, congenital abnormalities, chronic heart failure, a heart defect and cardiovascular infection" (Table 1). The research included only original research in the English language and peer reviews. Unpublished dissertations, book chapters, and conference papers were excluded.

Criteria for including or excluding papers were determined a priori. Papers were considered for inclusion only if (a) they were written in full-text English language in a peer-reviewed journal; (b) they were published between 2003 and 31 December 2019; and (c) they included subjects with cardiovascular diseases, coronary heart disease, congestive heart failure, congenital heart diseases, and chronic heart failure. Studies in which the sample presented with risk factors for the development of cardiovascular diseases (for example, pre-hypertension, hypertension, obesity, smoke, etc.) were also considered. Articles were excluded if (a) they considered subjects with a history of other neurological or psychiatric disorders; or (b) they considered other meditative practices such as transcendental meditation, autogenic training, or yoga. 
Table 1. Keywords and example of Search strategy.

\begin{tabular}{|c|c|c|c|}
\hline MeSH Terms & PubMed & Science Direct & Scholar \\
\hline “Heart Diseases" AND “mindfulness" & $11 / 15$ & $4 / 1187$ & $7 / 35,900$ \\
\hline “Cardiovascular Diseases" AND “Mindfulness" & $23 / 33$ & 0/1934 & $23 / 34,900$ \\
\hline "Heart Failure" AND "Mindfulness" & $1 / 2$ & $9 / 2168$ & $1 / 36,500$ \\
\hline "Cardiovascular failure and Mindfulness" & - & $6 / 1123$ & $5 / 24,200$ \\
\hline "Congestive heart failure and Mindfulness" & 3 & $3 / 444$ & $2 / 6510$ \\
\hline “Coronary Disease" AND “Mindfulness" & $3 / 3$ & $0 / 79$ & $11 / 20,500$ \\
\hline $\begin{array}{l}\text { “Cardiovascular Abnormalities" AND } \\
\text { “Mindfulness"; Congenital Abnormalities" } \\
\text { AND "Mindfulness"; "Heart Defects, } \\
\text { Congenital” AND “Mindfulness;" } \\
\text { Cardiovascular Infections" AND "Mindfulness" }\end{array}$ & - & $3 / 621$ & - \\
\hline
\end{tabular}

The data collected from each article were categorized as information on the first author and year of publication, the size of cohorts, the modalities of intervention, the type of clinical disorders, the study design, the outcomes (physical and psychological), the main results, and conclusions.

Following the Cochrane guidelines, the Quality Assessment of Diagnostic Accuracy Studies (QUADAS) tool [19] was used to assess the methodological quality and the risk of bias of each study. This quality assessment allowed classifying studies as having a low, high, or unknown risk of bias.

\section{Meta-Analyses}

Additional inclusion criteria were considered for the meta-analyses: (1) Participants should not overlap among studies; (2) studies reporting an explicit intervention protocol should be tested against a control group; (3) studies should study a population with comparable ages with the ones reported in at least 2 other studies.

Inclusion criteria used to select measures and constructs to analyze among the ones reported by the included studies were: (1) Outcome measures should have been taken $<1$ month after the study ended; (2) a construct or related measures should have been explored by at least 3 studies; (3) in studies with more than 2 groups, only MBI and control groups were selected.

Separate meta-analyses were conducted for each construct; the uncorrected $p$-value was set at 0.05 but was to be lowered in accordance with Sidak correction for multiple discoveries depending on the number of constructs that were included in the study in the end. Hedges' $g$ was used as an estimate of effect size.

Outcome measures were homogenized among studies so that positive $g$-values are always positive outcomes.

Even if we used additional criteria for the meta-analyses, we expected heterogeneity in treatment effects caused by differences in study populations (such as the age of patients), interventions received (such as online or in person), length of treatment, and other factors. Therefore, we chose a random-effects meta-analysis model because we assumed that the observed estimates of treatment effect can vary across studies due to the real differences in the treatment effect in each study and not only to sampling variability (chance) as assumed by the fixed model. We ran the fixed model anyway and report the data for completeness. Hedges' $g$ was used as an estimate of effect size. The $I$ square $\left(I^{2}\right)$ statistic represents the percentage of variability in effect estimates that is due to heterogeneity. The $I^{2}$ index can be interpreted as the percentage of total variability in a set of effect sizes due to true heterogeneity (between-studies variability). [20] In random-effects meta-analysis, the extent of variation among the effects observed in different studies (between-study variance) is referred to as tau-squared $\left(\tau^{2}\right)$ [20]. $\tau^{2}$ is the variance of the effect size parameters across the population of studies, and it reflects the variance of the true effect sizes. 


\section{Results}

\subsection{Study Selection}

The electronic bibliographic database search strategy of five databases retrieved 5600 studies. We screened titles/abstracts and adjusted for duplicates, and $N=115$ studies remained after the reviewing process. From this group, 61 studies were further excluded because they focused on other physical and/or psychological conditions. In the second phase, 54 studies were excluded because they did not fulfill inclusion criteria. Indeed, 24 studies used other meditative practices (such as yoga and transcendental meditation), and 13 studies were excluded for comorbidity with other physical and psychological conditions. Finally, 17 articles were included in this review (Figure 1).

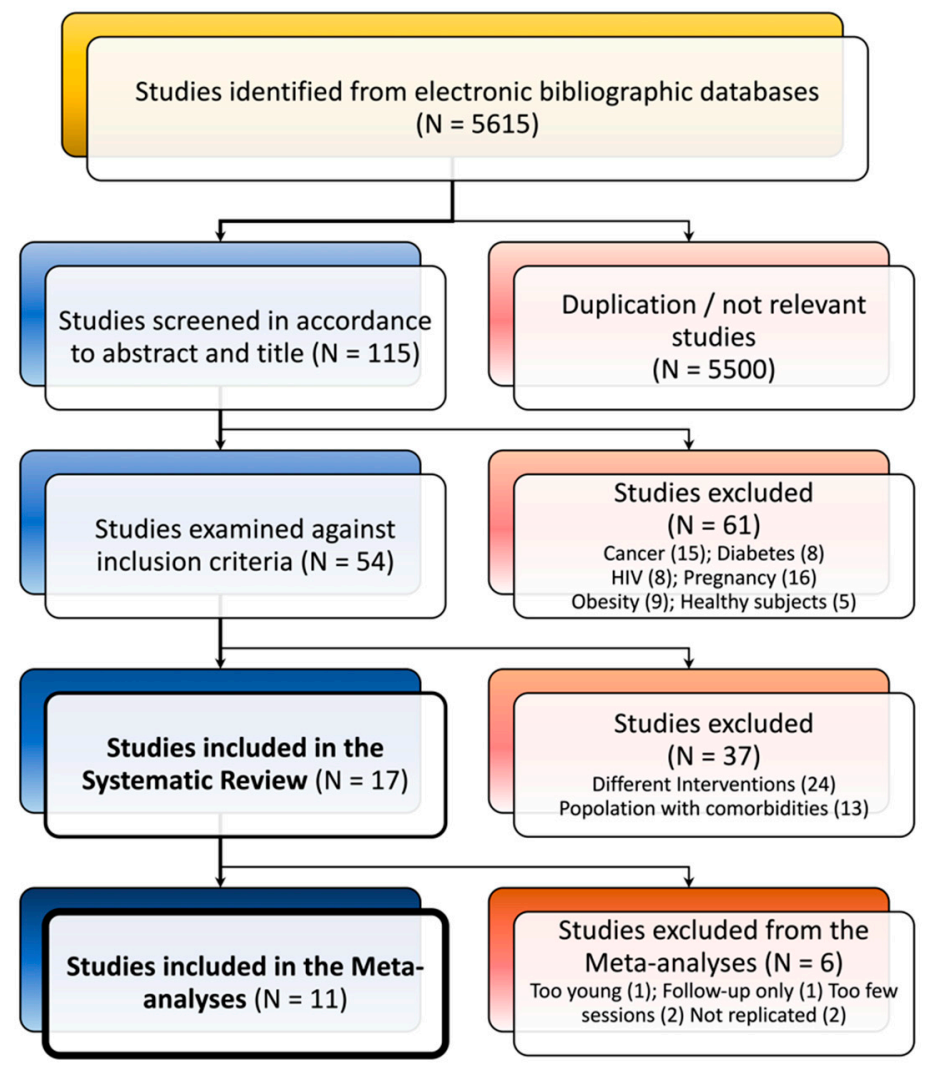

Figure 1. Flowchart of studies' identification.

\subsection{Study and Sample Characteristics}

The trial size ranged between 45 and 382 participants, with 5 out of 24 trials having $>100$ participants. The mean age of participants recruited ranged between 12 and 76 years. In total, 1611 participants were enrolled across 17 trials, and 16 out of 17 studies reported the gender of participants (women vs. men). Among the studies, 13 recruited both male and female participants, while 2 reported a sample of only female participants and 1 study of only males. All studies $(\mathrm{N}=17)$ examined a control group (waitlist or a different mindfulness intervention) (Table 2).

Six studies are reported in the review but were removed in the meta-analysis due to exclusion criteria. One study with teenage participants (mean age $=14.5$ ) [21] was excluded because the participant age was too different than the others. Another was a 12-month follow-up [22] of an already included study [17], 2 studies were a single section mindfulness experimental study to measure instant hearth measure changes $[23,24]$, and 2 studies used outcome measures taken by less than 2 other studies [25]. The 11 studies selected for meta-analysis reported 18 different constructs, but only 6 were reported in 3 or more studies. Selected constructs were anxiety, depression, perceived stress, quality of life, 
and measured blood pressure (diastolic and systolic). Each outcome was measured by 4 to 7 studies. The $p$-value was set to 0.009 (Table 3).

\subsection{Mindfulness and Physical Outcomes}

All studies confirmed the effectiveness of MBI-related treatments on the physical outcomes of CVD patients.

Five studies measured blood pressure. There was a large reduction in systolic blood pressure $\left(g=1.12,95 \%\right.$ CI: $\left.0.40 ; 1.84, p=0.002, I^{2}=89.1 \%, \tau^{2}=0.56\right)$, while diastolic blood pressure was not significant after correction $(g=0.66,95 \%$ CI: $-0.04 ; 1.36, p=0.043$, $\left.I^{2}=89.3 \%, \tau^{2}=0.54\right)[9,17,26-28]$ (Table 4, Figure 2).

Furthermore, one study also reported a significant reduction in heart palpitations $(\mathrm{F}=14.4, p<0.001)$ and heart rate (effect size, beats per minute: $-2.8,95 \% \mathrm{CI}$ : -5.4 ; $-0.2, p=0.033)$. Significant improvements were further detected in physical risk factors such as fatigue (effect-size using FSS sum-change on the group $=-8.0 ; p=0.0165$ ), unsteadiness/dizziness ( $p=0.039)$, breathlessness, and/or tiredness $(p=0.0087)$ [29].

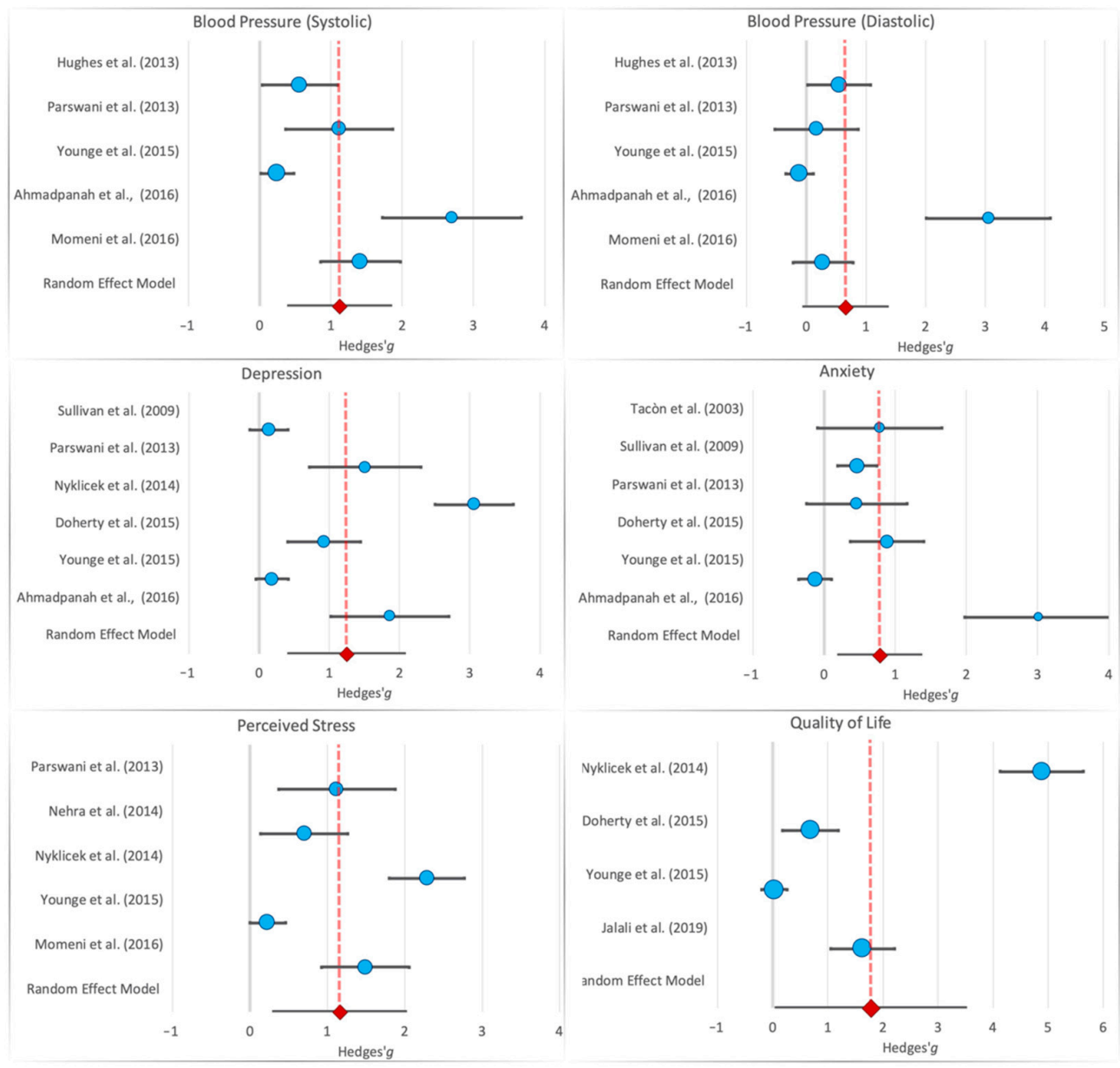

Figure 2. Forest plot for the six selected constructs. Dotted line is the Hedges' $g$ for the random-effects model $[9,10,17,26-28,30-33]$.

With regard to online mindfulness training, few studies have investigated the effects of this methodology on physical parameters $[17,21,22]$. Two studies showed a small positive effect on exercise capacity $(d=0.22 ; 95 \%$ CI $0.05 ; 0.39 ; p=0.055)$ and heart rate (beats per minute: $-2.8,95 \%$ CI: $-5.4 ;-0.2, p=0.033)[17,22]$. 
Table 2. Characteristics of studies assessing the effects of mindfulness on patients with cardiovascular disease.

\begin{tabular}{|c|c|c|c|c|c|c|c|}
\hline Reference & Sample & Country & Intervention & $\begin{array}{l}\text { Cardiological } \\
\text { Disorders }\end{array}$ & $\begin{array}{l}\text { Physiological } \\
\text { Measures }\end{array}$ & $\begin{array}{c}\text { Psychological } \\
\text { Measures }\end{array}$ & Main Findings \\
\hline $\begin{array}{l}\text { Ahmadpanah } \\
\text { et al. (2016) } \\
\text { [9] }\end{array}$ & $\begin{array}{l}\text { MDM ( } n 15 \text { female; Age: } \\
46.3 \text { year) } \\
\text { SMT ( } n 15 \text { female; Age: } \\
46.5 \text { year) } \\
\text { Control Group ( } n 15 \text { female; } \\
\text { Age: } 46.5 \text { year) }\end{array}$ & IRAN & $\begin{array}{l}\text { MDM group: } 8 \text { weekly sessions; } \\
\text { lasting } 1 \mathrm{~h} \\
\text { STM group: } 8 \text { weekly sessions, } \\
\text { lasting } 1 \mathrm{~h} \\
\text { Control Group: brief medical } \\
\text { check once a week for } 8 \text { weeks }\end{array}$ & HT & $\mathrm{BP}$ & $\begin{array}{l}\text { BDI } \\
\text { BAI }\end{array}$ & $\begin{array}{l}\text { MDM and SMT } \\
\text { treatments produced } \\
\text { improvements in } \\
\text { symptoms of anxiety } \\
\text { and depression and } \\
\text { reduced BP }\end{array}$ \\
\hline $\begin{array}{l}\text { Doherty et al. } \\
\text { (2015) [10] }\end{array}$ & $\begin{array}{l}\text { Treatment Group: } n 32 \\
\text { (12 women and } 19 \text { men; } \\
\text { mean age: } 57.6 \text { year) } \\
\text { Control Group: } 30 \\
\text { ( } 8 \text { women and } 22 \text { men mean } \\
\text { age: } 59.6 \text { year) }\end{array}$ & IRELAND & $\begin{array}{l}\text { MBCT group: } 8 \text { weekly sessions; } \\
\text { each lasted } 2 \mathrm{~h} \\
\text { Control Group: waiting list. } \\
\text { Received no psychological } \\
\text { intervention until Time } 3 \text {, when } \\
\text { they were offered MBCT. } \\
\text { All participants were assessed at } \\
\text { baseline (Time 1), } 8 \text { weeks later } \\
\text { (Time 2), and at 6-month } \\
\text { follow-up (Time 3) }\end{array}$ & $\mathrm{CHT}$ & & $\begin{array}{l}\text { HADS } \\
\text { BSI } \\
\text { POMS } \\
\text { PAIS } \\
\text { MAAS } \\
\text { PQ } \\
\text { CSQ }\end{array}$ & $\begin{array}{l}\text { MCBT showed } \\
\text { significant } \\
\text { improvement rates in } \\
\text { clinical depression. }\end{array}$ \\
\hline $\begin{array}{l}\text { Gotink et al. } \\
(2017)^{*} \\
\text { (follow-up) } \\
\text { [22] }\end{array}$ & $\begin{array}{l}\text { MBSR group: ( } n 215 ; \\
44.2 \% \text { female and } 55.8 \% \\
\text { male; mean age: } 43.2 \text { year) } \\
\text { Control Group: ( } n 109 ; \\
50.5 \% \text { female and } \\
49.5 \% \text { male; mean Age: } \\
43.2 \text { year) }\end{array}$ & JAPAN & $\begin{array}{l}\text { MBSR group: } 12 \text { weekly } \\
\text { sessions online } \\
\text { Control Group: usual care by } \\
\text { their treating cardiologist }\end{array}$ & Heart disease & $\begin{array}{l}\text { BP } \\
\text { Heart rate } \\
\text { Respiratory rate } \\
\text { Cortisol level }\end{array}$ & $\begin{array}{l}\text { SFHS } \\
\text { Visual Analogue } \\
\text { Scale } \\
\text { Hospital Anxiety and } \\
\text { Depression scale } \\
\text { Perceived Stress Scale } \\
\text { Perceived Social } \\
\text { Support Scale }\end{array}$ & $\begin{array}{l}\text { Exercise capacity, } \\
\text { systolic blood } \\
\text { pressure, mental } \\
\text { functioning, and } \\
\text { depression improved } \\
\text { significantly } \\
\text { compared to UC }\end{array}$ \\
\hline
\end{tabular}


Table 2. Cont.

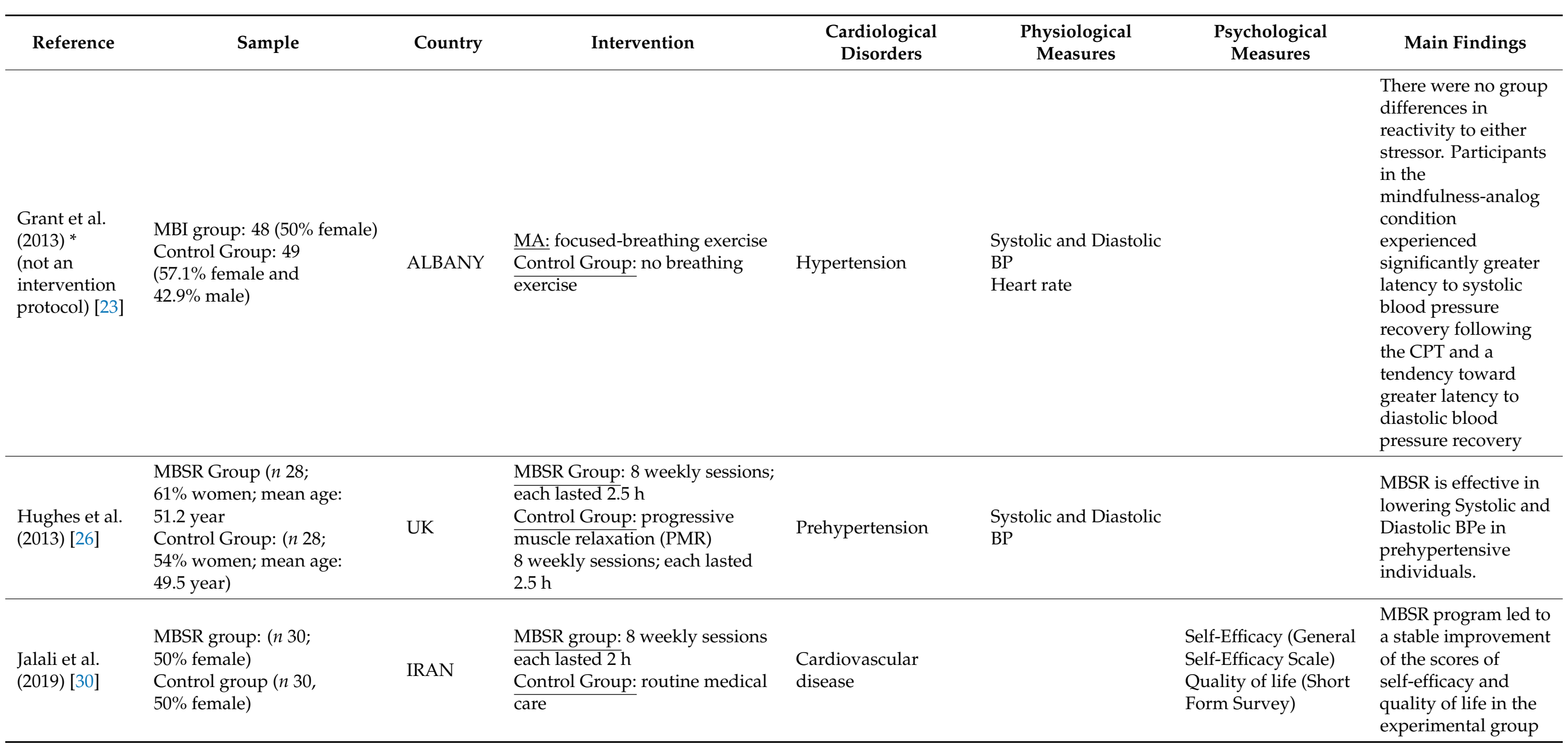


Table 2. Cont

\begin{tabular}{|c|c|c|c|c|c|c|c|}
\hline Reference & Sample & Country & Intervention & $\begin{array}{l}\text { Cardiological } \\
\text { Disorders }\end{array}$ & $\begin{array}{c}\text { Physiological } \\
\text { Measures }\end{array}$ & $\begin{array}{c}\text { Psychological } \\
\text { Measures }\end{array}$ & Main Findings \\
\hline $\begin{array}{l}\text { Momeni et al. } \\
\text { (2016) [28] }\end{array}$ & $\begin{array}{l}\text { MBRS group ( } n 30 \\
13 \text { female and } 17 \text { male Age: } \\
49.2 \text { year) } \\
\text { Control Group ( } n 30 \\
12 \text { female and } 18 \text { male; Age: } \\
46.2 \text { year }\end{array}$ & IRAN & $\begin{array}{l}\text { MBSR group: } 8 \text { weekly sessions } \\
\text { lasting } 2.5 \mathrm{~h} \\
\text { Control Group: receive no } \\
\text { psychological therapy, waiting } \\
\text { list }\end{array}$ & $\begin{array}{l}\text { Cardiovascular } \\
\text { disease }\end{array}$ & $\begin{array}{l}\mathrm{BP} \\
\mathrm{AOBP}\end{array}$ & $\begin{array}{l}\text { PSS-14 } \\
\text { STAI-X }\end{array}$ & $\begin{array}{l}\text { MBSR helps people } \\
\text { to deal with stress, } \\
\text { pain, and illness } \\
\text { more effectively and } \\
\text { play a more active } \\
\text { role in their lives and } \\
\text { recovery. MBSR was } \\
\text { also effective in } \\
\text { reducing cardiac } \\
\text { patients' BP, } \\
\text { perceived stress, and } \\
\text { anger. }\end{array}$ \\
\hline $\begin{array}{l}\text { Nehra et al. } \\
\text { (2014) [13] }\end{array}$ & $\begin{array}{l}\text { MBRS group ( } n \text { 25) } \\
\text { Control Group ( } n \text { 25) }\end{array}$ & INDIA & $\begin{array}{l}\text { MBSR group: } 8 \text { weekly sessions } \\
\text { lasting } 2 \mathrm{~h} \\
\text { Control Group: Standard } \\
\text { treatment }\end{array}$ & $\begin{array}{l}\text { Coronary heart } \\
\text { disease }\end{array}$ & & $\begin{array}{l}\text { PSS } \\
\text { HCS }\end{array}$ & $\begin{array}{l}\text { MBSR is highly } \\
\text { effective in reducing } \\
\text { perceived stress and } \\
\text { health complaints in } \\
\text { CHD patients. }\end{array}$ \\
\hline $\begin{array}{l}\text { Norman et al. } \\
(2018)[29]^{*} \\
\text { (not yet } \\
\text { replicated } \\
\text { measures) }\end{array}$ & $\begin{array}{l}\text { MBI group: } n 22 \text { (11 female } \\
\text { and } 11 \text { male; Mean Age: } \\
76.5 \text { year) } \\
\text { Control Group: } n 18 \\
\text { (6 female and } 12 \text { male; Mean } \\
\text { Age: } 75.0 \text { year) }\end{array}$ & Sweden & $\begin{array}{l}\text { MBI group: 8-weekly } \\
\text { educational and training } \\
\text { sessions lasting } 2 \mathrm{~h} \\
\text { Control group: received usual } \\
\text { care comprising standard health } \\
\text { care for patients with CHF }\end{array}$ & $\mathrm{CHF}$ & $\begin{array}{l}\text { Heart rate } \\
\text { Respiratory rate } \\
\text { Fatigue severity scale } \\
\text { Karolinska Sleep } \\
\text { Questionnaire } \\
\text { Unsteadiness/dizziness }\end{array}$ & & $\begin{array}{l}\text { MBI is effective in } \\
\text { reducing the } \\
\text { self-reported impact } \\
\text { of fatigue on daily } \\
\text { living, unsteadi- } \\
\text { ness/dizziness, and } \\
\text { breathless- } \\
\text { ness/tiredness } \\
\text { related to physical } \\
\text { function }\end{array}$ \\
\hline
\end{tabular}


Table 2. Cont

\begin{tabular}{|c|c|c|c|c|c|c|c|}
\hline Reference & Sample & Country & Intervention & $\begin{array}{l}\text { Cardiological } \\
\text { Disorders }\end{array}$ & $\begin{array}{c}\text { Physiological } \\
\text { Measures }\end{array}$ & $\begin{array}{c}\text { Psychological } \\
\text { Measures }\end{array}$ & Main Findings \\
\hline $\begin{array}{l}\text { Nyklicek et al. } \\
(2014)[31]\end{array}$ & $\begin{array}{l}\text { MBRS group } n 55 \text { (10 female } \\
\text { and } 45 \text { male; Mean Age: } \\
55.4 \text { year) } \\
\text { Mindfulness Self-Help } \\
\text { Control Group } n 52 \text { ( } 9 \text { female } \\
\text { and } 43 \text { male; Mean Age: } \\
56.3 \text { year) }\end{array}$ & USA & $\begin{array}{l}\text { MBSR: group: } 3 \text { weekly } \\
\text { meetings (lasting 90-120 min) } \\
\text { with an additional evaluation } \\
\text { session } \\
2 \text { weeks later). } \\
\text { Mindfulness Self-Help Control } \\
\text { Group: Participants receiving } \\
\text { the self-help booklet were asked } \\
\text { to thoroughly read the theory } \\
\text { and to practice exercises daily }\end{array}$ & PCI & & $\begin{array}{l}\text { SAD-4 } \\
\text { PSS } \\
\text { GMS } \\
\text { The World Health } \\
\text { Organization Quality } \\
\text { of Life-Brief } \\
\text { Questionnaire } \\
\text { SAQ } \\
\text { FMI-s }\end{array}$ & $\begin{array}{l}\text { The group } \\
\text { mindfulness } \\
\text { intervention reduced } \\
\text { perceived stress and } \\
\text { symptoms of anxiety } \\
\text { and depression more } \\
\text { strongly than the } \\
\text { control group in the } \\
\text { relatively younger } \\
\text { subsample. }\end{array}$ \\
\hline $\begin{array}{l}\text { Owens et al. } \\
(2016)[25]{ }^{*} \\
\text { (not yet } \\
\text { replicated } \\
\text { measures) }\end{array}$ & $\begin{array}{l}17 \text { female and } 3 \text { male; Mean } \\
\text { Age: } 49.4 \text { year }\end{array}$ & USA & $\begin{array}{l}\text { MBSR group: } 8 \text { weekly sessions } \\
\text { lasting } 2.5 \mathrm{~h} \\
\text { Control Group: Waiting list } \\
\text { control }\end{array}$ & Palpitations & Heart palpitations & & $\begin{array}{l}\text { MBSR participants } \\
\text { reported a significant } \\
\text { reduction in heart } \\
\text { palpitations, and this } \\
\text { improvement in the } \\
\text { MBSR participants } \\
\text { was sustained at } \\
\text { 1-month follow-up. }\end{array}$ \\
\hline $\begin{array}{l}\text { Parswani et al. } \\
\text { (2013) [27] }\end{array}$ & $\begin{array}{l}\text { MBSR group }(n 15 ; \text { male } \\
\text { mean age }=47.3 \text { year }) \\
\text { TAU group ( } n 15 \text { male mean } \\
\text { age }=50.6 \text { year })\end{array}$ & INDIA & $\begin{array}{l}\text { MBS group: eight weekly } \\
\text { sessions, lasting } 1-1.5 \mathrm{~h} \\
\text { Control Group: health } \\
\text { education session }\end{array}$ & $\mathrm{CHD}$ & $\begin{array}{l}\mathrm{BP} \\
\mathrm{BMI}\end{array}$ & $\begin{array}{l}\text { Hospital Anxiety and } \\
\text { Depression Scale } \\
\text { PSS }\end{array}$ & $\begin{array}{l}\text { MBSR treatment } \\
\text { produces significant } \\
\text { reduction in } \\
\text { symptoms of anxiety } \\
\text { and depression, } \\
\text { perceived stress, BP, } \\
\text { and BMI in CHD } \\
\text { patients }\end{array}$ \\
\hline
\end{tabular}


Table 2. Cont

\begin{tabular}{|c|c|c|c|c|c|c|c|}
\hline Reference & Sample & Country & Intervention & $\begin{array}{l}\text { Cardiological } \\
\text { Disorders }\end{array}$ & $\begin{array}{c}\text { Physiological } \\
\text { Measures }\end{array}$ & $\begin{array}{c}\text { Psychological } \\
\text { Measures }\end{array}$ & Main Findings \\
\hline $\begin{array}{l}\text { Steffen et al. } \\
(2015)[24]{ }^{*} \\
\text { (not an } \\
\text { intervention } \\
\text { protocol) }\end{array}$ & $\begin{array}{l}\text { MBI group: } n 30 \text { ( } 47 \% \text { female } \\
\text { and } 53 \% \text { male Age: } \\
19.9 \text { year) } \\
\text { Control Group: } n 32(53 \% \\
\text { female and } 47 \% \text { male Age: } \\
20.6 \text { year) }\end{array}$ & USA & $\begin{array}{l}\text { MBI group: Brief 1-day passive } \\
\text { listening of two tracks from } \\
\text { CD + Mindfulness of Breathing } \\
\text { exercise } \\
\text { Control Group: Brief 1-day } \\
\text { passive listening of two tracks } \\
\text { from CD }\end{array}$ & $\begin{array}{l}\text { Cardiovascular } \\
\text { reactivity }\end{array}$ & $\begin{array}{l}\mathrm{BP} \\
\text { Heart rate }\end{array}$ & $\begin{array}{l}\text { BDI } \\
\text { STAI } \\
\text { PASAT } \\
\text { Working memory } \\
\text { and auditory } \\
\text { attention }\end{array}$ & $\begin{array}{l}\text { Mindfulness } \\
\text { participants showed } \\
\text { lower systolic blood } \\
\text { pressure and } \\
\text { decreased systolic } \\
\text { during cognitively } \\
\text { stressing activity, } \\
\text { whereas no } \\
\text { significant effects } \\
\text { were detected for } \\
\text { mood levels. }\end{array}$ \\
\hline $\begin{array}{l}\text { Sullivan et al. } \\
\text { [32] (2009) }\end{array}$ & $\begin{array}{l}\text { MBSR group: } n 108 \text { ( } 66.7 \% \\
\text { male and } 33.3 \% \text { female Age: } \\
61.5 \text { year) } \\
\text { Control Group: } n 100(73 \% \\
\text { male and } 27 \% \text { female Age: } \\
61.1 \text { year) }\end{array}$ & UK & $\begin{array}{l}\text { MBSR group: once a week for } \\
2.25 \mathrm{~h} \text { for } 8 \text { consecutive weeks. } \\
\text { Control Group: Usual care } \\
\text { treatment }\end{array}$ & $\mathrm{CHF}$ & KCCQ 23-item & $\begin{array}{l}\text { CES-D 10-item } \\
\text { POMS }\end{array}$ & $\begin{array}{l}\text { MBSR significantly } \\
\text { reduced depression } \\
\text { and anxiety, while } \\
\text { improving overall } \\
\text { quality of life and } \\
\text { clinical symptoms in } \\
\text { patients with CHF } \\
\text { when compared to } \\
\text { control patients. }\end{array}$ \\
\hline $\begin{array}{l}\text { Tacòn et al. } \\
\text { (2003) [33] }\end{array}$ & $\begin{array}{l}\text { MBSR group: } n 10 \text { (female } \\
\text { Age: } 60.5 \text { year } \\
\text { Control Group: } n 10 \text { female } \\
\text { Age: } 60.5 \text { year }\end{array}$ & USA & $\begin{array}{l}\text { MBSR group: } 8 \text { weeks- } 2 \text { h each } \\
\text { week } \\
\text { Control Group: Participants } \\
\text { were placed on a waiting list } \\
\text { and offered the opportunity to } \\
\text { participate in the program after } \\
\text { the study was completed. }\end{array}$ & $\begin{array}{l}\text { Angina, } \\
\text { hypertension, } \\
\text { cardiovascular } \\
\text { disease, and cardiac } \\
\text { valve disorders. }\end{array}$ & & $\begin{array}{l}\text { STAI } \\
\text { Courtauld Emotional } \\
\text { Control Scale } \\
\text { Problem-Focused } \\
\text { Styles of Coping } \\
\text { Multidimensional } \\
\text { Health Locus of } \\
\text { Control scale }\end{array}$ & $\begin{array}{l}\text { Women in the } \\
\text { intervention group } \\
\text { showed } \\
\text { improvement in } \\
\text { anxiety scores and } \\
\text { decrease in the } \\
\text { control of negative } \\
\text { emotions. }\end{array}$ \\
\hline
\end{tabular}


Table 2. Cont.

\begin{tabular}{|c|c|c|c|c|c|c|c|}
\hline Reference & Sample & Country & Intervention & $\begin{array}{l}\text { Cardiological } \\
\text { Disorders }\end{array}$ & $\begin{array}{c}\text { Physiological } \\
\text { Measures }\end{array}$ & $\begin{array}{l}\text { Psychological } \\
\text { Measures }\end{array}$ & Main Findings \\
\hline $\begin{array}{l}\text { Younge et al. } \\
\text { (2015) [17] }\end{array}$ & $\begin{array}{l}\text { MBI group: } n 215 \\
\text { (44.2\% female and } 55.8 \% \\
\text { male Age: } 43.2 \text { year) } \\
\text { Control Group: } n 109 \\
\text { (50.5\% female and } 49.5 \text { male } \\
\text { Age: } 43.2 \text { year) }\end{array}$ & UK & $\begin{array}{l}\text { MBI group: 12-week session } \\
\text { lasting } 1 \mathrm{~h} \\
\text { Control group: usual care by } \\
\text { their treating cardiologist. } \\
\text { Treatment and frequency differ } \\
\text { between patients }\end{array}$ & Heart disease & $\begin{array}{l}\text { Heart rate } \\
\text { BP } \\
\text { Respiratory rate } \\
\text { NT-proBNP }\end{array}$ & $\begin{array}{l}\text { The Dutch version of } \\
\text { the Perceived Stress } \\
\text { Scale } \\
\text { Social Support Scale } \\
12 \text { Blumenthal }\end{array}$ & $\begin{array}{l}\text { Online mindfulness } \\
\text { training shows } \\
\text { positive effects on } \\
\text { heart rate, systolic } \\
\text { and diastolic blood } \\
\text { pressure. Whereas no } \\
\text { significant effect was } \\
\text { found on } \\
\text { psychological } \\
\text { outcomes. }\end{array}$ \\
\hline
\end{tabular}

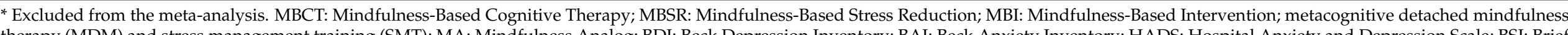

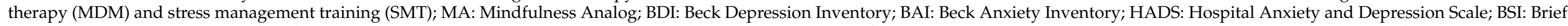

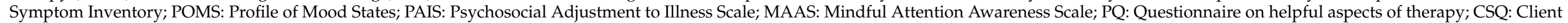

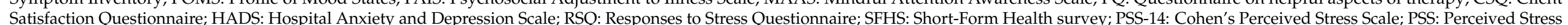

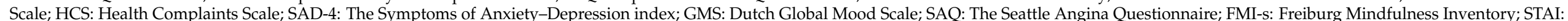

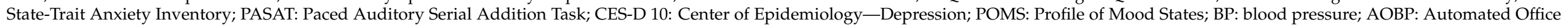

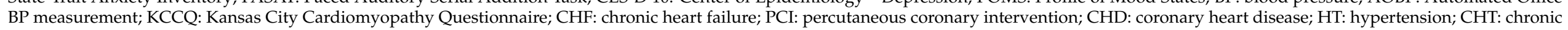
heart disease. 
Table 3. Statistical results for studies included in the meta-analysis.

\begin{tabular}{|c|c|c|c|c|c|c|c|c|c|c|c|c|c|c|}
\hline \multirow{3}{*}{$\begin{array}{r}\text { Construct } \\
\text { Anxiety }\end{array}$} & \multirow{3}{*}{$\begin{array}{c}\text { Study } \\
\text { Tacòn et al. (2003) [33] }\end{array}$} & \multicolumn{3}{|c|}{ MBI Group } & \multicolumn{3}{|c|}{ Control Group } & \multirow{3}{*}{\begin{tabular}{c} 
Cohen's \\
\multicolumn{1}{c}{} \\
0.814
\end{tabular}} & \multicolumn{4}{|c|}{ Hedges' $g$} & \multirow{3}{*}{$\begin{array}{r}\begin{array}{c}\text { Weight (\%) } \\
\text { Fixed Model }\end{array} \\
3.26\end{array}$} & \multirow{3}{*}{$\begin{array}{c}\begin{array}{c}\text { Weight (\%) } \\
\text { Random Model }\end{array} \\
14.1\end{array}$} \\
\hline & & \multirow{2}{*}{$\begin{array}{c}\mathbf{N} \\
10\end{array}$} & \multirow{2}{*}{\begin{tabular}{|c|} 
MD \\
-8.77
\end{tabular}} & \multirow{2}{*}{$\begin{array}{c}\text { SD } \\
9.31\end{array}$} & \multirow{2}{*}{$\begin{array}{c}\mathbf{N} \\
10\end{array}$} & \multirow{2}{*}{$\begin{array}{l}\text { MD } \\
0.330\end{array}$} & \multirow{2}{*}{$\begin{array}{c}\text { SD } \\
12.8\end{array}$} & & \multirow{2}{*}{$\begin{array}{c}g \\
0.779\end{array}$} & \multirow{2}{*}{$\begin{array}{c}\text { SE } \\
0.446\end{array}$} & \multicolumn{2}{|c|}{$95 \% \mathrm{CI}$} & & \\
\hline & & & & & & & & & & & -0.094 & 1.65 & & \\
\hline & Sullivan et al. (2009) [32] & 108 & -1.25 & 8.16 & 100 & 1.80 & 4.14 & 0.466 & 0.464 & 0.140 & 0.190 & 0.739 & 33.0 & 19.8 \\
\hline & Parswani et al. (2013) [27] & 15 & -1.74 & 3.30 & 15 & -0.140 & 3.49 & 0.471 & 0.458 & 0.360 & -0.248 & 1.16 & 5.00 & 15.8 \\
\hline & Doherty et al. (2015) [10] & 32 & -5.37 & 4.07 & 30 & -1.63 & 4.30 & 0.894 & 0.883 & 0.263 & 0.367 & 1.40 & 9.36 & 17.8 \\
\hline & Younge et al. (2015) [17] & 215 & -0.500 & 3.20 & 109 & -0.900 & 3.00 & -0.128 & -0.127 & 0.117 & -0.357 & 0.103 & 47.0 & 20.1 \\
\hline & Ahmadpanah et al. (2016) [9] & 15 & -6.20 & 4.57 & 15 & 7.87 & 4.54 & 3.09 & 3.01 & 0.526 & 1.98 & 4.04 & 2.34 & 12.5 \\
\hline \multirow{5}{*}{ Depression } & Parswani et al. (2013) [27] & 15 & -2.80 & 1.82 & 15 & 0.54 & 2.44 & 1.55 & 1.51 & 0.405 & 0.714 & 2.30 & 3.74 & 15.5 \\
\hline & Nyklicek et al. (2014) [31] & 55 & -1.61 & 0.450 & 52 & -0.210 & .460 & 3.08 & 3.06 & 0.284 & 2.51 & 3.62 & 7.61 & 16.7 \\
\hline & Doherty et al. (2015) [10] & 32 & -5.49 & 3.67 & 30 & -1.73 & 4.36 & 0.936 & 0.924 & 0.264 & 0.406 & 1.44 & 8.79 & 16.9 \\
\hline & Younge et al. (2015) [17] & 215 & -0.50 & 2.90 & 109 & 0.000 & 2.30 & 0.184 & 0.184 & 0.118 & -0.047 & 0.414 & 44.5 & 17.7 \\
\hline & Ahmadpanah et al. (2016) [9] & 15 & -8.00 & 4.52 & 15 & 1.33 & 5.22 & 1.91 & 1.86 & 0.429 & 1.02 & 2.70 & 3.34 & 15.2 \\
\hline \multirow[t]{4}{*}{$\begin{array}{r}\text { Perceived } \\
\text { Stress }\end{array}$} & Parswani et al. (2013) [27] & 15 & -10.5 & 4.13 & 15 & -2.74 & 8.58 & 1.16 & 1.13 & 0.384 & 0.373 & 1.88 & 5.68 & 18.6 \\
\hline & Nehra et al. (2014) [13] & 25 & -3.16 & 2.62 & 25 & -0.920 & 3.60 & 0.712 & 0.701 & 0.287 & 0.138 & 1.26 & 10.1 & 19.9 \\
\hline & Nyklicek et al. (2014) [31] & 55 & -4.5 & 1.04 & 52 & -2.05 & 1.05 & 2.30 & 2.29 & 0.248 & 1.80 & 2.77 & 13.7 & 20.3 \\
\hline & Momeni et al. (2016) [28] & 30 & -13.5 & 7.57 & 30 & -4.04 & 4.60 & 1.51 & 1.49 & 0.289 & 0.926 & 2.06 & 10.0 & 19.8 \\
\hline \multirow[t]{4}{*}{ Quality of Life } & Nyklicek et al. (2014) [31] & 55 & -2.55 & 0.370 & 52 & -0.630 & .410 & 4.92 & 4.88 & 0.385 & 4.13 & 5.64 & 6.37 & 24.4 \\
\hline & Doherty et al. (2015) [10] & 32 & -10.3 & 16.15 & 30 & 1.90 & 18.9 & 0.693 & 0.685 & 0.258 & 0.178 & 1.191 & 14.2 & 25.1 \\
\hline & Younge et al. (2015) [17] & 215 & 0.40 & 10.40 & 109 & 0.700 & 9.30 & 0.030 & 0.030 & 0.117 & -0.200 & 0.260 & 68.6 & 25.5 \\
\hline & Jalali et al. (2019) [30] & 30 & -13.0 & 8.35 & 30 & 0.970 & 8.59 & 1.65 & 1.63 & 0.295 & 1.05 & 2.21 & 10.8 & 24.9 \\
\hline \multirow{5}{*}{$\begin{array}{r}\text { Blood Pressure } \\
\text { (Diastolic) }\end{array}$} & Hughes et al. (2013) [26] & 28 & -2.40 & 5.30 & 28 & 1.10 & 7.11 & 0.558 & 0.550 & 0.269 & 0.024 & 1.08 & 12.3 & 20.9 \\
\hline & Parswani et al. (2013) [27] & 15 & -2.56 & 5.34 & 15 & -1.60 & 5.50 & 0.177 & 0.172 & 0.356 & -0.525 & 0.870 & 6.98 & 19.2 \\
\hline & Younge et al. (2015) [17] & 215 & -2.34 & 8.90 & 109 & -3.39 & 10.1 & -0.113 & -0.112 & 0.117 & -0.342 & 0.118 & 64.2 & 23.1 \\
\hline & Ahmadpanah et al. (2016) [9] & 15 & -21.5 & 10.7 & 15 & 9.69 & 9.17 & 3.14 & 3.051 & 0.530 & 2.01 & 4.09 & 3.14 & 15.6 \\
\hline & Momeni et al. (2016) [28] & 30 & -1.66 & 8.39 & 30 & 0.50 & 6.65 & 0.285 & 0.282 & 0.256 & -0.220 & 0.784 & 13.48 & 21.15 \\
\hline \multirow{5}{*}{$\begin{array}{r}\text { Blood Pressure } \\
\text { (Systolic) }\end{array}$} & Hughes et al. (2013) [26] & 28 & -4.90 & 6.87 & 28 & -0.70 & 7.83 & 0.570 & 0.562 & 0.269 & 0.035 & 1.089 & 12.68 & 20.98 \\
\hline & Parswani et al. (2013) [27] & 15 & -11.20 & 11.40 & 15 & 10.14 & 23.77 & 1.145 & 1.114 & 0.383 & 0.363 & 1.865 & 6.24 & 18.78 \\
\hline & Younge et al. (2015) [17] & 215 & -5.17 & 14.50 & 109 & -1.50 & 15.50 & 0.247 & 0.247 & 0.118 & 0.016 & 0.477 & 66.14 & 23.10 \\
\hline & Ahmadpanah et al. (2016) [9] & 15 & -36.33 & 15.59 & 15 & 9.53 & 17.46 & 2.771 & 2.696 & 0.497 & 1.721 & 3.671 & 3.70 & 16.46 \\
\hline & Momeni et al. (2016) [28] & 30 & -15.83 & 7.73 & 30 & -2.83 & 10.25 & 1.432 & 1.413 & 0.286 & 0.854 & 1.973 & 11.23 & 20.68 \\
\hline
\end{tabular}

MBI: Mindfulness-Based Intervention; N: number of participants; MD: mean difference between post-intervention and pre-intervention; SD: standard deviation; CI: confidence Interval. 
Table 4. Summary of meta-analysis results—fixed and random-effects models.

\begin{tabular}{|c|c|c|c|c|c|c|c|c|c|c|c|c|}
\hline \multirow{2}{*}{ Construct } & \multirow{2}{*}{ N Studies } & \multirow{2}{*}{ Model } & \multicolumn{6}{|c|}{ Hedges' g } & \multicolumn{4}{|c|}{ Heterogeneity } \\
\hline & & & $g$ & SE & \multicolumn{2}{|c|}{$95 \%$ CI } & $z$-Score & $p$-Value & $I^{2}$ & $x^{2}$ & $\tau^{2}$ & $d f$ \\
\hline \multirow[t]{2}{*}{ Anxiety } & 6 & Fixed & 0.295 & 0.081 & 0.137 & 0.453 & 3.66 & $<0.001 *$ & 0.894 & 47.34 & - & 5 \\
\hline & & Random & 0.780 & 0.294 & 0.204 & 1.38 & 2.65 & $0.008 *$ & 0.890 & - & 0.42 & - \\
\hline \multirow[t]{2}{*}{ Depression } & 6 & Fixed & 0.559 & 0.078 & 0.405 & 0.713 & 7.13 & $<0.001 *$ & 0.956 & 113.6 & - & 5 \\
\hline & & Random & 1.24 & 0.421 & 0.419 & 2.07 & 2.96 & 0.003 & 0.966 & - & 0.98 & - \\
\hline \multirow[t]{2}{*}{ Perceived Stress } & 5 & Fixed & 0.737 & 0.091 & 0.557 & 0.916 & 8.05 & $<0.001$ * & 0.939 & 65.47 & - & 4 \\
\hline & & Random & 1.16 & 0.435 & 0.306 & 2.01 & 2.66 & $0.007^{*}$ & 0.939 & - & 0.87 & - \\
\hline \multirow{2}{*}{ Quality of Life } & 4 & Fixed & 0.605 & 0.097 & 0.415 & 0.796 & 6.23 & $<0.001 *$ & 0.981 & 159.6 & - & 3 \\
\hline & & Random & 1.78 & 0.878 & 0.060 & 3.500 & 2.03 & 0.043 & 0.981 & - & 3.0 & - \\
\hline \multirow{2}{*}{$\begin{array}{l}\text { Blood Pressure } \\
\text { (Diastolic) }\end{array}$} & 5 & Fixed & 0.141 & 0.094 & -0.043 & 0.325 & 1.50 & 0.133 & 0.893 & 37.38 & . & 4 \\
\hline & & Random & 0.657 & 0.357 & -0.043 & 1.36 & 1.84 & 0.066 & 0.893 & - & 0.54 & - \\
\hline \multirow{2}{*}{$\begin{array}{l}\text { Blood Pressure } \\
\text { (Systolic) }\end{array}$} & 5 & Fixed & 0.563 & 0.096 & 0.375 & 0.750 & 5.88 & $<0.001$ * & 0.891 & 36.54 & - & 4 \\
\hline & & Random & 1.12 & 0.365 & 0.404 & 1.84 & 3.07 & $0.002 *$ & 0.891 & - & 0.56 & - \\
\hline
\end{tabular}




\subsection{Mindfulness and Psychological Outcomes}

MBI was effective in reducing mood symptomatology and perception of stress across a variety of clinical populations $[9,10,13,22,24,27,28,30,31,33]$. Only one study did not describe any significant psychological benefit before and after MBI intervention [17].

The main psychological benefit concerns depression symptomatology. There was a very large effect of MBI on depression among 6 studies included in the meta-analysis $\left(g=1.24,95 \%\right.$ CI: $\left.0.42 ; 2.07, p=0.003, I^{2}=95.6 \%, \tau^{2}=0.98\right)[9,10,17,27,31,32]$ (Table 4, Figure 2).

O' Doherty et al. [10] demonstrated that depressed patients with CVD who completed an 8-session MBCT training had an effect size for depressive symptoms of $d=0.77$ after treatment and $d=0.60$ at the 6-month follow-up.

Five studies used perceived stress as an outcome measure. In such studies, a significantly large effect of mindfulness in CVD was observed for perceived stress $(g=1.16$, 95\% CI: $\left.0.31 ; 2.01, p=0.007, I^{2}=93.9 \%, \tau^{2}=0.87\right)[13,17,27,28,31]$. Six studies used anxiety as an outcome variable. In such studies, a significantly moderate effect of mindfulness in CVD was also observed $\left(g=0.78,95 \%\right.$ CI: $0.20 ; 1.36, p=0.008, I^{2}=89.4 \%$, $\left.\tau^{2}=0.42\right)[9,10,17,27,32,33]$ (Table 4, Figure 2). This effect was also observed in adolescents $(t(1,9)=3.67, p<0.01)[34$ ] and partially in young adults $(t(105)=1.84, p=0.07)$ [31]. Similarly, in older adults, there was a significant reduction in anxiety between pre- and post-intervention $(\mathrm{F}(1,16)=6.79, p<0.01 ; t=6.14, p<0.001 ; t=4.16, p<0.001$-respectively, Tacón et al. [33], Sullivan et al. [32], Parswani et al. [27]). Furthermore, Keyworth et al. [35] reported a reduction in thoughts linked with worries $(t(37)=4.39, p<0.001, d=0.42)$. A significant difference between the groups in terms of patients' perceived stress was also found in other studies $(\mathrm{F}=107.62, p<0.001 ; t(1,25)=2.47, p<0.001 ; t(1,102)=3.10$, $p=0.002$ - respectively, Momeni et al. [28], Nehra et al. [13], and Nyklíček et al. [31]).

Quality of life was reported by four studies. The summary effect size was very large, but in the random-effects model, it was not significant due to excessive variability among studies $\left(g=1.78,95 \%\right.$ CI: $\left.0.06 ; 3.50, p=0.043, I^{2}=98.1 \%, \tau^{2}=3.0\right)[10,17,30,31]$ (Table 4, Figure 2).

Other studies reported significant improvements in a variety of psychological factors, such as emotional control, coping strategies, and self-efficacy. For instance, Tacón et al. [33] revealed that participants of their mindfulness group showed an improvement in expressing negative feelings compared to pre-intervention $(F(1,16)=6.26, p<0.02)$. Freedenberg et al. [21] have found that some styles of coping strategies (i.e., cognitive restructuring, acceptance, positive thinking, and distraction) increased significantly from before to after mindfulness interventions $(\mathrm{F}(1,44)=5.191, p=0.028)$. Specifically, significant differences and changes in direction were found for the reactive style $(\mathrm{F}(1,16)=5.52, p=0.03)$. In relation to perceived stress, the authors [21] found a significant decrease in stress after both the MBSR and online interventions $(\mathrm{F}(1,43)=13.94, p=0.001)$. As concerns self-efficacy, research conducted by Jalali et al. [30] revealed no significant differences between experimental and control groups, whereas significant changes in self-efficacy and quality of life occurred after 3 months, at the follow-up assessment $(p<0.01)$ [30].

As for the use of the online version of mindfulness training, some studies showed a significant effect specifically on stress $(\mathrm{F}(1,43)=13.94, p=0.001)$ and coping $(\mathrm{F}(1,44)=5.91$, $p=0.028)$ [21], mental functioning $(d=0.22,95 \%$ CI $0.05 ; 0.38 ; p=0.108)$ and depressive symptomatology $(d=0.18,95 \%$ CI $0.02 ; 0.35 ; p=0.143)$ [22].

\subsection{Risk of Bias}

For some studies, the methods of randomization were unclear. Furthermore, few studies described group allocation and randomization. Some RCTs reported adequate random sequence generation and allocation concealment and adequate blinding of participants ${ }^{\prime}$ personnel and outcome assessment [26]. Only a few studies had a low risk of bias in all other criteria $[13,23]$. 


\section{Discussion}

All studies included in this systematic review described relevant effects of MBI intervention on physical and/or psychological outcomes of patients with CVD. For physical outcomes, what clearly emerged from the meta-analysis is that MBI intervention has a real benefit in reducing systolic blood pressure, and the literature review suggests that heart palpitations and rate could also decrease. MBI had a moderate effect size in the reduction of diastolic blood pressure, but the meta-analysis was not significant. Moreover, for some physical risk factors, such as the impact of fatigue, unsteadiness/dizziness, breathlessness, and/or tiredness, an additional significant impact was also detected by a study [29]. As concerns psychological outcomes (such as depression, anxiety, stress, quality of life, styles of coping), all studies confirmed that different MBI-related approaches positively impact overall psychological wellbeing. Some studies found significant statistical effects for combined psychological conditions $[9,13,27,28,30-33]$, whereas others found only a specific effect in one single domain, such as depression [10,22]. In contrast, Younge and colleagues [17] are the only ones who did not report significant differences before and after MBI in CVD patients. Nevertheless, the meta-analyses showed a moderate, large, and very large effect size for anxiety, perceived stress, and depression, respectively. As for quality of life, the random-effects model effect size was very large, but there were only four studies with a large variability; therefore, it was not significant.

Some researchers have highlighted how physiological measures (heart rate, blood pressure) can be influenced by mindfulness practice, suggesting significant improvements in pre- and post-treatment scores $[17,22,25-27,29]$. Furthermore, several studies have shown a direct correlation between mindfulness practice and scores related to psychological measures such as anxiety and depression $[9,10,22,27,31,32]$. The psychological wellbeing linked to mindfulness practice was also quantified in two studies that compared the scores relating to self-efficacy and perceived quality of life [29,30,32]. They showed an improvement in these aspects, thus highlighting the positive effects related to mindfulness on psychological wellbeing. However, Steffen et al. [24] and Younge et al. [17] did not report improvements in scores related to psychological measures, although better physiological outcomes (heart rate beat and blood pressure) were detected.

\section{Limitations}

Part of the discrepancy reported in this systematic re-examination of literature may be dependent on some factors, such as (a) the different duration of MBI intervention (from 1 day to 12 weeks) $[17,22]$, (b) the psychological battery, (c) the high dropout rates $[10,17,26]$, and (d) the administration system. One of the main aspects that could influence the outcome of CVD patients is the duration of MBI treatments. Indeed, a wide heterogeneity characterized the literature where MBI-related training may last from 2 to 12 weeks, without any specific reference. Cullen et al. [36] claimed that to obtain significant statistical results, the training should last at least 8 sessions. Hence, when a shorter protocol has been used, the results are unreliable statistically [23]. Additionally, some studies evaluated mindfulness skills with specific surveys and scales [10], while others did not use any objective evaluation $[23,25,26,29]$. Moreover, in some studies, the training was led by a certified trainer $[10,21,26,28,31]$, while in others, it was not $[9,13,17,22-25,27,29,30,32,33]$. Therefore, this aspect may have affected the impact of training on physical and psychological outcomes.

Another important aspect is the administration modality of MBI (in vivo vs. online). As regards online administration, there are few studies in the literature that have investigated this methodology $[17,21,22]$, and the results of these studies are controversial because some studies showed a small positive effect on psychological outcomes (stress, mental functioning, and depressive symptomatology) [21], whereas others did not $[17,22]$. As regards physical outcomes, the same studies demonstrated a significant improvement in exercise capacity, systolic blood pressure, and heart rate $[17,22]$. These controversial results may depend on several factors: the age of the participants (adults vs. teenagers) and the 
methodology used (videoconference vs. online program). In addition, another facet of online training must be considered: the lack of external control. Indeed, teachers cannot control whether participants have practiced the tasks accurately. This can lead to less motivation and lower adherence than training with teachers and other group members [17]. Considering the paucity of studies on this approach, it is still not possible to recommend it. Finally, even if almost all of the studies reported the gender of participants, gender was not actually used as a factor for outcome analysis. Therefore, future studies should analyze gender differences for this kind of intervention.

\section{Conclusions}

This review was planned and conducted following best practice guidelines for systematic reviews and meta-analyses. Many RCTs in the general population as well as in high-risk disease groups were included. Subgroup analyses were conducted to assess the effects among these different participant groups. The applicability of the results was assessed. From the examined studies, convergent results are found. Despite some methodological differences (i.e., a few studies described group allocation, adequate random sequence generation, the predominant presence of women), we can affirm that there is sufficient evidence to conclude that MBI can be included in clinical recommendations for managing CVD symptoms. In future studies, it would be better to evaluate the effectiveness of online MBI applications, mainly in the context of a pandemic such as COVID-19, where there is a need to avoid direct contact between clinicians and patients and to reduce the number of admissions in hospital. Indeed, a middle way would therefore be ideal: an easily accessible online training but with more content and feedback from a trainer. It will also be important to replicate physiological and psychological outcome measures not included in the meta-analyses while increasing the diversity of the setting, methods, and population to include them as moderators. Again, other important aspects remain to be established, such as the use of measures on mindfulness skills, teacher competency, and the adherence to the intervention protocol to disentangle therapist effects from intervention-content effects.

Author Contributions: Conceptualization, F.M., C.F., C.C., and G.P.; methodology, F.M., C.F., C.C., and S.G. (Sebastiano Gangemi); software, A.P., G.T., A.A.A., and D.V.; validation, F.M., C.F., P.C., R.M., G.T., L.R., and D.V.; formal analysis, A.A.A., S.G. (Sara Genovese), A.P., G.T., S.G. (Sebastiano Gangemi), D.V., A.C., and G.P.; investigation, F.M., C.F., C.C., and M.C.; resources, F.M., M.C., F.C., L.R., D.V., and G.P.; data curation, F.M., C.F., S.G. (Sara Genovese), F.C., D.V., S.G. (Sebastiano Gangemi), and A.A.A.; writing — original draft preparation, F.M., C.F., S.G. (Sebastiano Gangemi), and A.C.; writing - review and editing, F.M., F.C., S.G. (Sebastiano Gangemi), A.C., D.V., and G.P.; visualization, F.M., A.C., and G.P.; supervision, F.M., S.G. (Sebastiano Gangemi), A.C., and G.P.; project administration, G.P.; funding acquisition, G.P. All authors have read and agreed to the published version of the manuscript.

Funding: This research was funded by the Sicilian Region of Italy (Assessorato Regionale delle Attività Produttive), grant number n. 087490210350, entitled BRAINHEART-Sistemi intelligenti per la prevenzione delle malattie cardiovascolari nell'anziano attraverso il benessere psicofisico, P.O. F.E.S.R. Sicilia 2014/2020, Azione 1.1.5.

Institutional Review Board Statement: Not applicable.

Informed Consent Statement: Not applicable.

Conflicts of Interest: The authors declare no conflict of interest.

\section{References}

1. Mozaffarian, D.; Benjamin, E.J.; Go, A.S.; Arnett, D.K.; Blaha, M.J.; Cushman, M.; De Ferranti, S.; Després, J.P.; Fullerton, H.J.; Howard, V.J.; et al. Heart Disease and Stroke Statistics-2015 Update: A Report from the American Heart Association. Circulation 2015, 131, e29-e322. [CrossRef]

2. Loucks, E.B.; Britton, W.B.; Howe, C.J.; Eaton, C.B.; Buka, S.L. Positive Associations of Dispositional Mindfulness with Cardiovascular Health: The New England Family Study. Int. J. Behav. Med. 2015, 22, 540-550. [CrossRef] 
3. Tunstall-Pedoe, H.; Kuulasmaa, K.; Mähönen, M.; Tolonen, H.; Ruokokoski, E.; Amouyel, P. Contribution of trends in survival and coronary-event rates to changes in coronary heart disease mortality: 10-year results from 37 WHO MONICA Project populations. Lancet 1999, 353, 1547-1557. [CrossRef]

4. Lawrence, M.; Booth, J.; Mercer, S.; Crawford, E. A systematic review of the benefits of mindfulness-based interventions following transient ischemic attack and stroke. Int. J. Stroke 2013, 8, 465-474. [CrossRef] [PubMed]

5. Mars, T.S.; Abbey, H. Mindfulness meditation practise as a healthcare intervention: A systematic review. Int. J. Osteopath. Med. 2010, 13, 56-66. [CrossRef]

6. Kabat-Zinn, J. Wherever You Go, There You Are: Mindfulness Meditation for Everyday Life; Hyperion: New York, NY, USA, 1994.

7. Scott-Sheldon, L.A.J.; Gathright, E.C.; Donahue, M.L.; Balletto, B.; Feulner, M.M.; Decosta, J.; Cruess, D.G.; Wing, R.R.; Carey, M.P.; Salmoirago-Blotcher, E. Mindfulness-Based Interventions for Adults with Cardiovascular Disease: A Systematic Review and Meta-Analysis. Ann. Behav. Med. 2020, 54, 67-73. [CrossRef]

8. Kabat-Zinn, J. Full Catastrophe Living: Using the Wisdom of Your Body and Mind to Face Stress, Pain, and Illness; Random House Publishing Group: New York, NY, USA, 2013.

9. Ahmadpanah, M.; Paghale, S.J.; Bakhtyari, A.; Kaikhavani, S.; Aghaei, E.; Nazaribadie, M.; Holsboer-Trachsler, E.; Brand, S. Effects of psychotherapy in combination with pharmacotherapy, when compared to pharmacotherapy only on blood pressure, depression, and anxiety in female patients with hypertension. J. Health Psychol. 2016, 21, 1216-1227. [CrossRef] [PubMed]

10. O’Doherty, V.; Carr, A.; McGrann, A.; O’Neill, J.O.; Dinan, S.; Graham, I.; Maher, V. A Controlled Evaluation of Mindfulness-Based Cognitive Therapy for Patients with Coronary Heart Disease and Depression. Mindfulness 2015, 6, 405-416. [CrossRef]

11. Fogarty, F.A.; Booth, R.J.; Gamble, G.D.; Dalbeth, N.; Consedine, N.S. The effect of mindfulness-based stress reduction on disease activity in people with rheumatoid arthritis: A randomised controlled trial. Ann. Rheum. Dis. 2015, 74, 472-474. [CrossRef]

12. Labelle, L.E.; Campbell, T.S.; Faris, P.; Carlson, L.E. Mediators of Mindfulness-Based Stress Reduction (MBSR): Assessing the Timing and Sequence of Change in Cancer Patients. J. Clin. Psychol. 2015, 71, 21-40. [CrossRef]

13. Nehra, D.K.; Sharma, N.; Kumar, P.; Nehra, S. Efficacy of mindfulness-based stress reduction programme in reducing perceived stress and health complaints in patients with coronary heart disease. Dysphrenia 2014, 5, 19-25.

14. Morgan, D. Mindfulness-Based Cognitive Therapy for Depression: A New Approach to Preventing Relapse. Psychother. Res. 2003, 13, 123-125. [CrossRef]

15. Abbott, R.A.; Whear, R.; Rodgers, L.R.; Bethel, A.; Thompson Coon, J.; Kuyken, W.; Stein, K.; Dickens, C. Effectiveness of mindfulness-based stress reduction and mindfulness based cognitive therapy in vascular disease: A systematic review and meta-analysis of randomised controlled trials. J. Psychosom. Res. 2014, 76, 341-351. [CrossRef] [PubMed]

16. Alsubaie, M.; Abbott, R.; Dunn, B.; Dickens, C.; Keil, T.F.; Henley, W.; Kuyken, W. Mechanisms of action in mindfulness-based cognitive therapy (MBCT) and mindfulness-based stress reduction (MBSR) in people with physical and/or psychological conditions: A systematic review. Clin. Psychol. Rev. 2017, 55, 74-91. [CrossRef]

17. Younge, J.O.; Wery, M.F.; Gotink, R.A.; Utens, E.M.W.J.; Michels, M.; Rizopoulos, D.; Van Rossum, E.F.C.; Hunink, M.G.M.; Roos-Hesselink, J.W. Web-based mindfulness intervention in heart disease: A randomized controlled trial. PLoS ONE 2015, 10, e0143843. [CrossRef]

18. Moher, D.; Liberati, A.; Tetzlaff, J.; Altman, D.G.; Grp, P. Preferred Reporting Items for Systematic Reviews and Meta-Analyses: The PRISMA Statement (Reprinted from Annals of Internal Medicine). Phys. Ther. 2009, 89, 873-880. [CrossRef]

19. Reitsma, J.B.; Leeflang, M.M.G.; Sterne, J.A.C.; Bossuyt, P.M.M.; Whiting, P.F.; Rutjes, A.W.S.S.; Westwood, M.E.; Mallet, S.; Deeks, J.J.; Reitsma, J.B.; et al. QUADAS-2: A Revised Tool for the Quality Assessment of Diagnostic Accuracy Studies. Ann. Intern. Med. 2011, 155, 529-536.

20. Jonathan, J.; Deeks, J.P.H.; Altman, D.G. 9 Analysing Data and Undertaking Meta-Analyses; Jonathan, J., Deeks, J., Higgins, P.T., Altman, D.G., on behalf of the Cochrane Statistical Methods Group, Eds.; Wiley-Blackwell, John Wiley \& Sons Ltd.: Chichester, UK, 2008; ISBN 9780470699515.

21. Freedenberg, V.A.; Hinds, P.S.; Friedmann, E. Mindfulness-Based Stress Reduction and Group Support Decrease Stress in Adolescents with Cardiac Diagnoses: A Randomized Two-Group Study. Pediatr. Cardiol. 2017, 38, 1415-1425. [CrossRef] [PubMed]

22. Gotink, R.A.; Younge, J.O.; Wery, M.F.; Utens, E.M.W.J.; Michels, M.; Rizopoulos, D.; Van Rossum, L.F.C.; Roos-Hesselink, J.W.; Hunink, M.M.G. Online mindfulness as a promising method to improve exercise capacity in heart disease: 12-month follow-up of a randomized controlled trial. PLOS ONE 2017, 12, 0175923. [CrossRef]

23. Grant, C.; Hobkirk, A.; Persons, E.; Hwang, V.; Danoff-Burg, S. Cardiovascular reactivity to and recovery from stressful tasks following a mindfulness analog in college students with a family history of hypertension. J. Altern. Complement. Med. 2013, 19, 341-346. [CrossRef]

24. Steffen, P.R.; Larson, M.J. A Brief Mindfulness Exercise Reduces Cardiovascular Reactivity During a Laboratory Stressor Paradigm. Mindfulness 2015, 6, 803-811. [CrossRef]

25. Owens, J.E.; Schorling, J.; Plews-Ogan, M.; Goodman, M.; Moorman, R.; Zaklin, R.; Dent, J. A randomized controlled trial evaluating Mindfulness-Based Stress Reduction (MBSR) for the treatment of palpitations: A pilot study. Int. J. Cardiol. 2016, 223, 25-27. [CrossRef] [PubMed]

26. Hughes, J.W.; Fresco, D.M.; Myerscough, R.; Van Dulmen, M.H.M.; Carlson, L.E.; Josephson, R. Randomized controlled trial of mindfulness-based stress reduction for prehypertension. Psychosom. Med. 2013, 75, 721-728. [CrossRef] [PubMed] 
27. Parswani, M.; Sharma, M.; Iyengar, S. Mindfulness-based stress reduction program in coronary heart disease: A randomized control trial. Int. J. Yoga 2013, 6, 111. [CrossRef]

28. Momeni, J.; Omidi, A.; Raygan, F.; Akbari, H. The effects of mindfulness-based stress reduction on cardiac patients' blood pressure, perceived stress, and anger: A single-blind randomized controlled trial. J. Am. Soc. Hypertens. 2016, 10, 763-771. [CrossRef]

29. Norman, J.; Fu, M.; Ekman, I.; Björck, L.; Falk, K. Effects of a mindfulness-based intervention on symptoms and signs in chronic heart failure: A feasibility study. Eur. J. Cardiovasc. Nurs. 2018, 17, 54-65. [CrossRef] [PubMed]

30. Jalali, D.; Abdolazimi, M.; Alaei, Z.; Solati, K. Effectiveness of mindfulness-based stress reduction program on quality of life in cardiovascular disease patients. IJC Heart Vasc. 2019, 23, 100356. [CrossRef]

31. Nyklíček, I.; Dijksman, S.C.; Lenders, P.J.; Fonteijn, W.A.; Koolen, J.J. A brief mindfulness based intervention for increase in emotional well-being and quality of life in percutaneous coronary intervention (PCI) patients: The MindfulHeart randomized controlled trial. J. Behav. Med. 2014, 37, 135-144. [CrossRef]

32. Sullivan, M.J.; Wood, L.; Terry, J.; Brantley, J.; Charles, A.; McGee, V.; Johnson, D.; Krucoff, M.W.; Rosenberg, B.; Bosworth, H.B.; et al. The Support, Education, and Research in Chronic Heart Failure Study (SEARCH): A mindfulness-based psychoeducational intervention improves depression and clinical symptoms in patients with chronic heart failure. Am. Heart J. 2009, 157, 84-90. [CrossRef]

33. Tacón, A.M.; McComb, J.; Caldera, Y.; Randolph, P. Mindfulness meditation, anxiety reduction, and heart disease: A pilot study. Fam. Community Health 2003, 26, 25-33. [CrossRef] [PubMed]

34. Freedenberg, V.A.; Thomas, S.A.; Friedmann, E. A Pilot Study of a Mindfulness Based Stress Reduction Program in Adolescents with Implantable Cardioverter Defibrillators or Pacemakers. Pediatr. Cardiol. 2015, 36, 786-795. [CrossRef] [PubMed]

35. Keyworth, C.; Knopp, J.; Roughley, K.; Dickens, C.; Bold, S.; Coventry, P. A mixed-methods pilot study of the acceptability and effectiveness of a brief meditation and mindfulness intervention for people with diabetes and coronary heart disease. Behav. Med. 2014, 40, 53-64. [CrossRef] [PubMed]

36. Cullen, M. Mindfulness-Based Interventions: An Emerging Phenomenon. Mindfulness 2011, 2, 186-193. [CrossRef] 